\title{
The Feasibility Analysis of Using A New Network Architecture to Solve the Tidal Effects
}

\author{
HOU Weina ${ }^{1, a}$, CAO Xian-wei ${ }^{2, b}$, LIU Zhan-jun ${ }^{3,}$ \\ $1,2,3$ Key Lab of Mobile Communication Technology, Chongqing university of Posts and \\ Telecommunications, Chongqing 400065, China \\ ahouwnj@cqupt.edu.cn, bvanas@163.com, cliuzj@cqupt.edu.cn
}

Keywords: RAN, new network architecture, tidal effects,feasibility

\begin{abstract}
To solve high cost, high energy and many other issues in the communication, propose a new network architecture, one of the important applications is to solve the "tidal effect", by BBUs uniform placement to achieve unified deployment of resources to get the purpose of saving computation. But its feasibility has not been verified, this paper will verify its feasibility by data from two aspects of changing the number of base stations and changing the different situations of the base stations. Be measured through simulation and analysis results show that the use of new network architecture can not only save computation, but also enhance the resource utilization and reduce construction, operation and maintenance costs.
\end{abstract}

\section{Introduction}

Radio Access Network (RAN) is the main development direction of communications now, The infrastructure of operators are becoming increasingly sophisticated. However, in the environment of rapid development of mobile services and the increasingly intense competitive, mobile operators face many challenges: the high energy consumption, rising construction and operation maintenance costs, tight spectrum, the rapid growth of flow and increasingly severe cost pressures.

In order to solve these problems, reference[1] proposed a new network architecture that relies on the "BBU + RRU" base structure. the RRUs, covering different regions, via fiber optic connection to the BBUs, the baseband resources located in the BBUs, shared by the all RRUs. BBUs as a "baseband pool", in control of all of the baseband resources, distribute baseband resources according to the various regions of the traffic. Telephone traffic can share the same baseband resources when they in different regions; when some base resource fails, also select the resources of "baseband pool" as a backup, to improve system stability.

A specific application of this architecture of the wireless communication is to solve tidal effects. A inherent characteristics of the network is that the user is on the move, according to a long-term observation, the user's mobile shows a significant trends following the time. As these mobile users, mobile network load also shows the phenomenon of migration in the network with time. In a traditional radio access network, the processing power of each base station can only be used for the users within the district of their service. When the user leaves the district, the processing power of base station can not be transferred, only wasted. As the operators need to maintain network coverage all times, so that these no-load or small load base stations must consume the same power with the high-load power station, and because the user segment in a moment together in some areas, the base station often at full capacity, the need for processing power is much higher than average. Therefore, on the one side, there is a waste of processing power, the other is the lack of processing power.

\section{System model}

Traditional network architecture. In the traditional network architecture, the distribution of each base station is independent and its resources used alone. Assume that the number of simultaneous calls is random for single base station at each moment, there can assume at time ${ }^{t}$, the same time 
the volume of business $\mathrm{X}$ to meet the normal distribution $F(X)$ with the probability of occurrence, with the changes of $t$, the mathematical expectation $\mu(t)$ and variance $\sigma(t)$ are a function of $t$, so can get a random process. When there are $N$ base stations, you can get $N$ normal random process of time $t$ and volume of business at this time, the probability density function are:

$$
f_{n}(x, t)=\frac{1}{\sigma_{n}(t) \sqrt{2 \pi}} \exp \left\{-\frac{\left(x-\mu_{n}(t)\right)^{2}}{2 \sigma_{n}^{2}(t)}\right\} \quad(n=1,2,3 \ldots \ldots . N)
$$

Where $x$ is the volume of business for the moment $t, f_{n}$ is generated probability density of the business volume of the base station at time ${ }^{t}$.

Assuming $\mu_{i}(t)=g_{i}(t)$ and $\sigma_{i}(t)=\xi_{i}(t)$ for any base station $i$,in order to make the base station can handle business accounts for the volume of business that may be encountered as a percentage $P$ of the maximum, according to the relationship between probability density and distribution, can get the relationship:

$$
\int_{0}^{\varphi(t)} f_{i}(x, t) d x \geq P
$$

According to this formula can obtain the business volume of the base station $i$ to meet requirements of above-mentioned, according to the different value of $t$ can obtain the maximum $\varphi_{i}$ of $\varphi(t)$ available, namely the base station require to meet the volume of business in requirements of the above-mentioned conditions. When has $N$ Base stations, because calls between each base station independently of each other, so the total of base stations to meet the total volume of business is:

$$
\text { Total }=\sum_{i=1}^{N} \varphi_{i}
$$

New network architecture. After the application of new network architectures, BBUs unified place to form a dynamic resource pool, all the resources unified configuration through cloud computing. At time $t$, assume that the volume of business $X_{i}$ of each base station and its distribution $F_{i}(X)$ is consistent with traditional network architecture, but the resources of all base stations is configured uniformly, so all base stations form a joint distribution $F(y)$. At time $t$, the total volume of business of all base stations is $Y=X_{1}+X_{2}+\ldots \ldots+X_{N}$. Because business of each base station is independent, according to the available knowledge of probability theory can know: $Y \sim N\left(\mu_{1}(t)+\mu_{2}(t)+\ldots+\mu_{N}(t), \sigma_{1}^{2}(t)+\sigma_{2}^{2}(t)+\ldots+\sigma_{N}^{2}(t)\right)$. Assuming the probability density is:

$$
f(y, t)=\frac{1}{\sigma_{(t)}^{\prime} \sqrt{2 \pi}} \exp \left\{-\frac{\left(y-\mu_{(t)}^{\prime}\right)^{2}}{2 \sigma_{(t)}^{\prime 2}}\right\}
$$

Where $\mu_{(t)}^{\prime}=\mu_{1}(t)+\mu_{2}(t)+\ldots+\mu_{N}(t)$ is mean, $\sigma_{(t)}^{\prime}=\sqrt{\sigma_{1}^{2}(t)+\sigma_{2}^{2}(t)+\ldots+\sigma_{N}^{2}(t)}$ is variance, $y$ is the total business volume of all base stations at time $t, f$ is the probability density when all base stations have ${ }^{y}$ volume of business at time ${ }^{t}$.

At this time, the percentage of total business of $N$ base stations to meet accounts the largest business of these base stations is $P$, According to the relationship between probability density and distribution can be obtained relationship

$$
\int_{0}^{\delta(t)} f(y, t) d y \geq P
$$

According to equation 5 and different values of $t$ can obtaine the maximum value $\delta$ of $\delta(t)$, so that all base stations required to meet the total volume of business is:

$$
\text { Total' }=\delta
$$


Comparison. Assuming base station need $Q$ calculation to deal with each business, by Section A and Section B can draw the traditional network architecture and new network architectures under the same conditions of the different computational requirements, this time can obtaine the difference of computation between the two architectures through the operation of calculating, that is:

$$
\text { Value }=\left(\text { Total }- \text { Total }{ }^{\prime}\right) Q
$$

When Value is greater than 0 , the new network architecture saves computation than traditional network architecture; when Value is equal to 0 , the two networks the same amount of computation required for the structure; when Value is less than 0 , the new network architecture than the traditional network architecture requires more computation.

\section{Computer simulation and analysis}

Computer evaluation. Based on the above model can obtaine the required data by setting the corresponding parameters. For any base station $i$, assuming its mean and variance is $\mu_{i}(t)$ and $\sigma_{i}(t)$ respectively.

$$
\begin{gathered}
\mu_{i}(t)=30 \sin \left(2 t / a_{i}-3\right)+c_{i}-t \\
\sigma_{i}(t)=2 \cos \left(t / a_{i}-2\right)+b_{i}
\end{gathered}
$$

Where $a_{i}, b_{i}, c_{i}$ are the random values generated by computer within a certain range, values are evenly distributed. As the distribution $F$ is normal distribution, direct solution would be more difficult, so can turn its form to a standard normal distribution, deform as:

$$
\varnothing_{i}\left(\frac{\varphi_{i}(t)-\mu_{i}(t)}{\sigma_{i}(t)}\right)=\int_{0}^{\frac{\varphi_{i}(t)-\mu_{i}(t)}{\sigma_{i}(t)}} \frac{1}{\sqrt{2 \pi}} \exp \left\{-\frac{x^{2}}{2}\right\} d x \geq P
$$

By the available knowledge of probability, the value of $\rho$ varies with the value $P$, can be obtained the value of $\varphi_{i}(t)$ varies with the value of time $t$ through the using of computer programming. Based on the model, the process flow diagram can be drawn as follows:

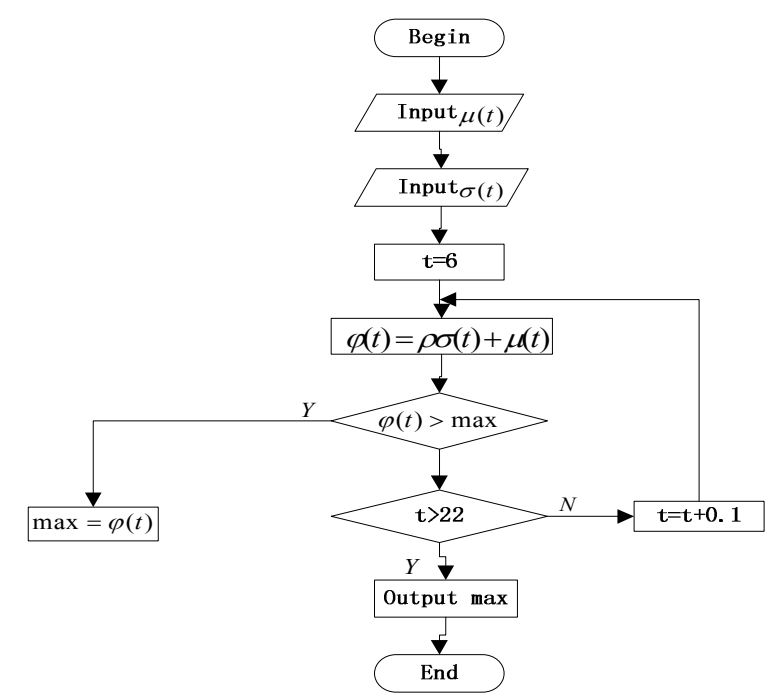

Figure 1 Evaluation program flow chart

A variable number of base stations. Different aspects can be evaluated on the amount of computation. Assuming that the percentage $P$ of total business of $N$ base stations to meet accounts the largest business of these base stations is fixed value, it can take $99 \%$, the amount of computation will be varied with the number of base stations changes. Based on the above, taking $N=9$ can get the datashown in table 1 . 
Table 1 Each base station to meet the volume of business

\begin{tabular}{|c|c|}
\hline Number of base station & Volume of business \\
\hline 1 & 101.18 \\
\hline 2 & 107.59 \\
\hline 3 & 95.84 \\
\hline 4 & 102.61 \\
\hline 5 & 70.81 \\
\hline 6 & 86.03 \\
\hline 7 & 105.92 \\
\hline 8 & 102.58 \\
\hline 9 & 114.17 \\
\hline
\end{tabular}

The value of Total and Total' change with the changing number of base stations, here can get the data throuhg procedures and simulation, shown in Figure 1.

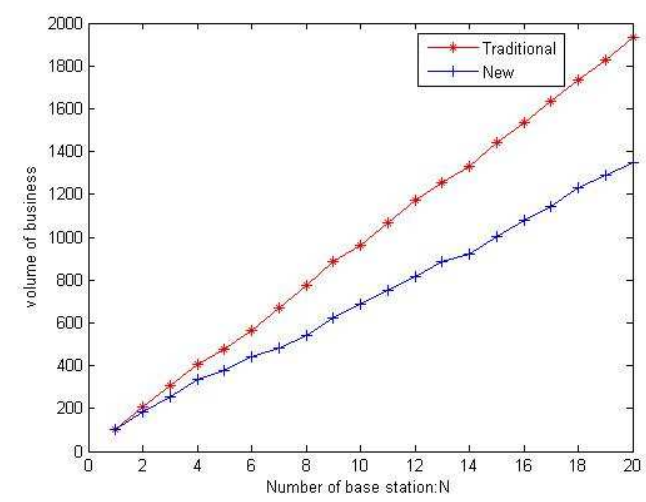

Figure 2 Business volume growth in the number of changes with the base station

Figure 2 shows that requirement of the volume of business for the two architectures is same when $P$ under certain conditions and number of base station is 1 . But with the increase in the number of base stations, The requirement of volume of business is increasing for new network architecture and traditional network architecture, and the increase of traditional network architecture is faster than new network architecture. This shows that the new network architecture is more saving computation than the traditional calculation, and with the base station increases, the savings becomes increasingly evident.

Percentage of variable. When the number of base stationa is stationary, the case of the base station to meet the different $P$, at this time it is another case. Assuming the number $N$ of base station has been determined as 20 , and $85 \%$ of the $P$ Provisional Acceptance, At this time the traditional network architecture required for each base station to meet the volume of business as follows:

In the case of 20 base stations, with the changes in $P$, the total volume of business is diffrernt between the traditional network architecture and new network architecture, can get figure 3 :

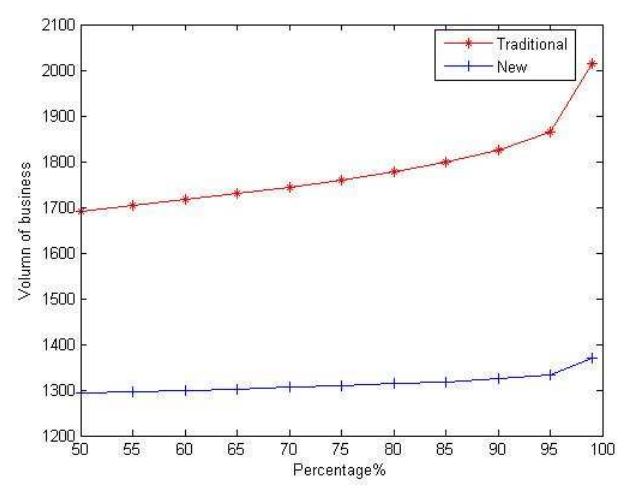

Figure 3 Volume of business with the percentage change in different 
Figure 3 shows that, in certain cases the number of base stations, with the increasing of percentage for the base station to meet, the volume of business of traditional network architectures and new network infrastructure required to meet are increasing. However, the requirement of new network architecture is less than traditional network architecture, and new network architecture has a lower growth rate. It indicates that, the new network architecture saves more computation than traditional network architecture, moreover, with the percentage increasing, this saving is more and more obviously.

\section{Conclusion}

It can be seen that the new network architecture has the obvious advantage than the traditional network architecture, the greater capacity of the baseband pool has, the higher efficiency of resource sharing capability and the more effective support to achieve TD baseband pool technologies.

Based resource sharing scheme has a very wide range of applications, in addition to used in the scenario which has significant traffic migration, such as metro, area, community. And also be used in scenarios of traffic-intensive and requiring high stability of the system, such as conference centers, large stadiums and so on. It can be said that baseband resource sharing scheme can be applied to cover the vast majority of scenes, it is essential solutions for building high-quality network.

\section{Acknowledgment}

This work is supported by the National Major Project of China under Grant No. 2012ZX03003008-4.

\section{References}

[1] Seppo Hamalainen. Self-Organizing Networks in 3GPP LTE[C]//Proceedings of 2009 International Conference on Vehicular Technology. Finland: IEEE, 2009:1-2

[2] 3GPP TS 32.500, Telecommunication management; Self-Organizing Networks (SON); Concepts and requirement. V11.1.0 [S].2011.12

[3] 3GPP TS 36.401, Evolved Universal Terrestrial Radio Access Network (E-UTRAN); Architecture description. V10.3.0 [S]. 2011.9

[4] 3GPP TS 32.150, Telecommunication management; Integration Reference Point (IRP) Concept and definitions. V10.2.0[S]. 2011.3

[5] 3GPP TS 32.541, Telecommunication management; Self-Organizing Networks (SON); Self-healing concepts and requirements. V10.0.0 [S].2011.3

[6] 3GPP TS 32.501, Telecommunication management; Telecommunication management; Self-configuration of network elements; Concepts and requirements. V10.0.0 [S]. 2011.3 